\title{
Some Results on the Vector Gaussian Hypothesis Testing Problem
}

\author{
Pierre Escamilla Abdellatif Zaidi ${ }^{\dagger} \ddagger \quad$ Michèle Wigger * \\ $\dagger$ Paris Research Center, Huawei Technologies, Boulogne-Billancourt, 92100, France \\ $\ddagger$ Université Paris-Est, Champs-sur-Marne, 77454, France \\ * LTCI, Télécom Paris, Université Paris-Saclay, Palaiseau, 91120, France \\ \{pierre.escamilla@gmail.com, abdellatif.zaidi@u-pem.fr\} \\ \{michele.wiggeretelecom-paristech.fr\}
}

\begin{abstract}
This paper studies the problem of discriminating two multivariate Gaussian distributions in a distributed manner. Specifically, it characterizes in a special case the optimal typeII error exponent as a function of the available communication rate. As a side-result, the paper also presents the optimal type-II error exponent of a slight generalization of the hypothesis testing against conditional independence problem where the marginal distributions under the two hypotheses can be different.
\end{abstract}

\section{INTRODUCTION}

Consider the single-sensor single-detector hypothesis testing scenario in Fig. 11 The sensor observes a source sequence $\mathbf{X}^{n} \triangleq\left(\mathbf{X}_{1}, \ldots, \mathbf{X}_{n}\right)$ and communicates with the detector, who observes source sequence $\mathbf{Y}^{n} \triangleq\left(\mathbf{Y}_{1}, \ldots, \mathbf{Y}_{n}\right)$, over a noise-free bit-pipe of rate $R \geq 0$. Here, $n$ is a positive integer that denotes the blocklength and the sequence of pairs $\left\{\left(\mathbf{X}_{t}, \mathbf{Y}_{t}\right)\right\}_{t=1}^{n}$ is independent and identically distributed (i.i.d) according to a jointly Gaussian distribution of zero-mean and of joint covariance matrix that depends on the hypothesis $\mathcal{H} \in\{0,1\}$. Under hypothesis

$$
\mathcal{H}=0:\left\{\left(\begin{array}{l}
\mathbf{X}_{t} \\
\mathbf{Y}_{t}
\end{array}\right)\right\}_{t=1}^{n} \text { i.i.d. } \sim \mathcal{N}(\mathbf{0}, \mathbf{K}),
$$

and under hypothesis

$$
\mathcal{H}=1:\left\{\left(\begin{array}{l}
\mathbf{X}_{t} \\
\mathbf{Y}_{t}
\end{array}\right)\right\}_{t=1}^{n} \text { i.i.d. } \sim \mathcal{N}(\mathbf{0}, \overline{\mathbf{K}}) .
$$

Based on its observations $\mathbf{Y}^{n}$ and the message it receives from the sensor, the Detector decides on the hypothesis by producing $\hat{\mathcal{H}} \in\{0,1\}$. The goal of this decision is to maximize the exponential decrease of the probability of type-II error (i.e., of guessing $\hat{\mathcal{H}}=0$ when $\mathcal{H}=1$ ), while ensuring that the probability of type-I error (i.e., guessing $\hat{\mathcal{H}}=1$ when $\mathcal{H}=0$ ) goes to zero as $n \rightarrow \infty$.

The described single-sensor single-detector problem has previously been studied in [1]-[4] for various joint distributions on the i.i.d. observations. In particular, [4] identified the largest type-II exponent that is achievable in a setup that they termed testing against conditional independence. An explicit expression for the vector Gaussian case was recently found in [5] (see Theorem 2 therein which actually provides the solution of a more general, distributed, setting). For all other cases a computable single-letter characterization of the largest achievable type-II error exponent remains open. This line of works has also been extended to multiple sensors [2], [4][6], multiple detectors [7], [8], interactive terminals [9]-[11], multi-hop networks [12]-[16], noisy channels [17], [18] and to scenarios with privacy constraints [19]-[21].

In this paper we present a computable single-letter characterization of the largest type-II error exponent achievable for the Gaussian vector hypothesis testing problem for a class of matrices $\mathbf{K}$ and $\overline{\mathbf{K}}$. Our converse proof starts from the known multi-letter expression for this problem [1] and connects it to related results. The achievability proof is based on the coding scheme proposed in [3].

We end this introductory section with some remarks on notation. When two random variables $(X, Y)$ are independent given a third random variable $Z$ (i.e. $P_{X Y Z}=P_{Z} P_{X \mid Z} P_{Y \mid Z}$ ), we say $(X, Z, Y)$ form a Markov chain and write $X \ominus Z \ominus Y$. Both $D\left(P_{X} \| P_{\bar{X}}\right)$ and $D(X \| \bar{X})$ denote the Kullback-Leiber divergence between two pmfs $P_{X}$ and $P_{\bar{X}} \cdot h(\cdot), I(\cdot ; \cdot)$ and $I(\cdot ; \cdot \cdot \cdot)$ denote continuous entropy, mutual information and conditional mutual information. The set of all real numbers is denoted by $\mathbb{R}$. Boldface upper case letters denote random vectors or deterministic matrices, e.g., $\mathbf{X}$, where the context should make the distinction clear. We denote the covariance matrix of a real-valued vector $\mathbf{X}$ with distribution $P_{\mathbf{X}}$ by $\mathbf{K}_{\mathbf{X}}=\mathbb{E}_{P_{\mathbf{X}}}\left[\mathbf{X X}^{\dagger}\right]$, where ${ }^{\dagger}$ indicates the transpose operation. Similarly, we denote the cross-correlation of two zero-mean vectors $\mathbf{X}$ and $\mathbf{Y}$ with joint distribution $P_{\mathbf{X Y}}$ by $\mathbf{K}_{\mathbf{X Y}}=\mathbb{E}_{P \mathbf{X Y}}\left[\mathbf{X Y} \mathbf{Y}^{\dagger}\right]$, the conditional covariance matrix of $\mathbf{X}$ given $\mathbf{Y}$ with p.d.f $P_{\mathbf{X Y}}$ and with p.d.f $\bar{P}_{\mathbf{X Y}}$ by $\mathbf{K}_{\mathbf{X} \mid \mathbf{Y}}=\mathbb{E}_{P_{\mathbf{X Y}}}\left[\mathbf{X X} \mathbf{X}^{\dagger} \mid \mathbf{Y}\right]$ and $\overline{\mathbf{K}}_{\mathbf{X} \mid \mathbf{Y}}=\mathbb{E}_{\bar{P}_{\mathbf{X Y}}}\left[\mathbf{X X} \mathbf{X}^{\dagger} \mid \mathbf{Y}\right]$, respectively. Finally, for a matrix $\mathbf{M}$, we denote its inverse (if it exists) by $\mathbf{M}^{-1}$ its determinant (if it exists) by $|\mathbf{M}|$, its MoorePenrose pseudo-inverse by $\mathbf{M}^{+}$and its pseudo-determinant by $|\mathbf{M}|_{+}$.

\section{FORMAL PROBLEM STATEMENT}

The sequences $\mathbf{X}^{n}$ and $\mathbf{Y}^{n}$ are as described before, where

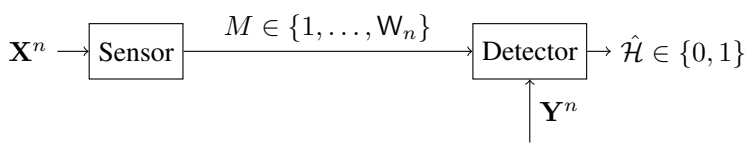

Fig. 1. Vector Gaussian hypothesis testing problem

we denote by $m$ the dimension of each vector $\mathbf{X}_{t}$ and by $q$ 
the dimension of each vector $\mathbf{Y}_{t}$. The Sensor, which observes $\mathbf{X}^{n}$ applies an encoding function

$$
\phi_{n}: \mathbb{R}^{m \times n} \rightarrow \mathcal{M}=\left\{1, \ldots, \mathrm{W}_{n}\right\}
$$

to this sequence and sends the resulting index

$$
M=\phi_{n}\left(\mathbf{X}^{n}\right)
$$

to the detector. Based on this message $M$ and its observation $\mathbf{Y}^{n}$, the detector then applies a decision function

$$
\psi_{n}: \mathcal{M} \times \mathbb{R}^{q \times n} \rightarrow\{0,1\}
$$

to decide on the hypothesis

$$
\hat{\mathcal{H}}=\psi_{n}\left(M, \mathbf{Y}^{n}\right) .
$$

The Type-I and type-II error probabilities at the detector are defined as:

$$
\begin{gathered}
\alpha_{n} \triangleq \operatorname{Pr}\{\hat{\mathcal{H}}=1 \mid \mathcal{H}=0\} \\
\beta_{n} \triangleq \operatorname{Pr}\{\hat{\mathcal{H}}=0 \mid \mathcal{H}=1\} .
\end{gathered}
$$

Definition 1. Given rate $R \geq 0$, an error-exponent $\theta$ is said achievable if for all blocklengths $n$ there exist functions $\phi_{n}$ and $\psi_{n}$ as in (3) and (5) so that the following limits hold:

$$
\begin{gathered}
\lim _{n \rightarrow \infty} \alpha_{n}=0, \\
\theta \leq \varliminf_{n \rightarrow \infty}-\frac{1}{n} \log \beta_{n}
\end{gathered}
$$

and

$$
\varlimsup_{n \rightarrow \infty} \frac{1}{n} \log _{2} \mathrm{~W}_{n} \leq R .
$$

Definition 2 (Exponent-rate function). For any rate $R \geq 0$, the exponent-rate function $E(R)$ is the supremum of the set of all achievable error-exponents.

In essence, the problem of vector Gaussian hypothesis testing that we study here amounts to discriminating two covariance matrices. As we already mentioned the solution of this problem is known only in few special cases, namely the cases of testing against independence and testing against conditional independence [1], [4], and [5, Theorem 2].

\section{OPTIMAL EXPONENT FOR A CLASS OF COVARIANCE MATRICES}

Let $\mathbf{K}_{\mathbf{X}}$ and $\overline{\mathbf{K}}_{\mathbf{X}}$ be $m$-by- $m$ dimensional matrices, $\mathbf{K}_{\mathbf{Y}}$ and $\overline{\mathbf{K}}_{\mathbf{Y}}$ be $q$-by- $q$ dimensional matrices, and $\mathbf{K}_{\mathbf{X Y}}$ and $\overline{\mathbf{K}}_{\mathbf{X Y}}$ be $m$-by- $q$ dimensional matrices such that

$$
\mathbf{K}=\left[\begin{array}{cc}
\mathbf{K}_{\mathbf{X}} & \mathbf{K}_{\mathbf{X Y}} \\
\mathbf{K}_{\mathbf{X Y}}^{\dagger} & \mathbf{K}_{\mathbf{Y}}
\end{array}\right] \text { and } \overline{\mathbf{K}}=\left[\begin{array}{cc}
\overline{\mathbf{K}}_{\mathbf{X}} & \overline{\mathbf{K}}_{\mathbf{X Y}} \\
\overline{\mathbf{K}}_{\mathbf{X Y}}^{\dagger} & \overline{\mathbf{K}}_{\mathbf{Y}}
\end{array}\right] \text {. }
$$

Further, define the condition $\mathrm{C}$ :

$$
\begin{aligned}
\mathbf{C :} \mathbf{K}_{\mathbf{X Y}}= & \underset{\mathbf{G}}{\arg \min } \log \mid\left[\begin{array}{cc}
\mathbf{I} & \mathbf{0} \\
\mathbf{0} & \overline{\mathbf{K}}_{\mathbf{X Y}} \overline{\mathbf{K}}_{\mathbf{Y}}^{-1}
\end{array}\right] \overline{\mathbf{K}}\left[\begin{array}{cc}
\mathbf{I} & \mathbf{0} \\
\mathbf{0} & \overline{\mathbf{K}}_{\mathbf{X Y}} \overline{\mathbf{K}}_{\mathbf{Y}}^{-1}
\end{array}\right]_{+}^{\dagger} \\
& -\log |\boldsymbol{\Gamma}| \\
& +\operatorname{Tr}\left\{\left(\left[\begin{array}{cc}
\mathbf{I} & \mathbf{0} \\
\mathbf{0} & \overline{\mathbf{K}}_{\mathbf{X Y}} \overline{\mathbf{K}}_{\mathbf{Y}}^{-1}
\end{array}\right] \overline{\mathbf{K}}\left[\begin{array}{cc}
\mathbf{I} & \mathbf{0} \\
\mathbf{0} & \overline{\mathbf{K}}_{\mathbf{X Y}} \overline{\mathbf{K}}_{\mathbf{Y}}^{-1}
\end{array}\right]^{\dagger}\right)^{+} \boldsymbol{\Gamma}\right\}
\end{aligned}
$$

where the minimum is over all $m$-by- $q$ matrices $\mathbf{G}$ such that the matrix

$$
\boldsymbol{\Gamma} \triangleq\left[\begin{array}{cc}
\mathbf{K}_{\mathbf{X}} & \mathbf{G}^{\dagger} \overline{\mathbf{K}}_{\mathbf{Y}}^{-1} \overline{\mathbf{K}}_{\mathbf{X Y}}^{\dagger} \\
\overline{\mathbf{K}}_{\mathbf{X Y}} \overline{\mathbf{K}}_{\mathbf{Y}}^{-1} \mathbf{G} & \overline{\mathbf{K}}_{\mathbf{X Y}} \overline{\mathbf{K}}_{\mathbf{Y}}^{-1} \mathbf{K}_{\mathbf{Y}} \overline{\mathbf{K}}_{\mathbf{Y}}^{-1} \overline{\mathbf{K}}_{\mathbf{X Y}}^{\dagger}
\end{array}\right] .
$$

is positive semi-definite, i.e.,

$$
\Gamma \succeq 0 \text {. }
$$

The following theorem provides an explicit analytic expression of the exponent-rate function of the vector Gaussian hypothesis testing problem of Figure 1 when condition $\mathrm{C}$ in (11) is fulfilled.

Theorem 1. If $\mathrm{C}$ is satisfied, then

$$
\begin{aligned}
E & (R) \\
= & \frac{m}{2}+\frac{q}{2}+\frac{1}{2} \log \frac{\left|\overline{\mathbf{K}}_{\mathbf{Y}}\right|}{\left|\mathbf{K}_{\mathbf{Y}}\right|}+\frac{1}{2} \operatorname{Tr}\left(\overline{\mathbf{K}}_{\mathbf{Y}}^{-1} \mathbf{K}_{\mathbf{Y}}\right) \\
+ & \frac{1}{2} \log \left|\overline{\mathbf{K}}_{\mathbf{X} \mid \mathbf{Y}}\right|-\log \mid \mathbf{K}_{\mathbf{X}}-\mathbf{K}_{\mathbf{X Y}} \overline{\mathbf{K}}_{\mathbf{Y}}^{-1} \\
& \times \overline{\mathbf{K}}_{\mathbf{X Y}}^{\dagger}\left(\overline{\mathbf{K}}_{\mathbf{X Y}} \overline{\mathbf{K}}_{\mathbf{Y}}^{-1} \mathbf{K}_{\mathbf{Y}} \overline{\mathbf{K}}_{\mathbf{Y}}^{-1} \overline{\mathbf{K}}_{\mathbf{X Y}}^{\dagger}\right)^{+} \overline{\mathbf{K}}_{\mathbf{X Y}} \overline{\mathbf{K}}_{\mathbf{Y}}^{-1} \mathbf{K}_{\mathbf{X Y}}^{\dagger} \mid \\
+ & \frac{1}{2} \operatorname{Tr}\left(\overline{\mathbf{K}}_{\mathbf{X} \mid \mathbf{Y}}^{-1} \times\right. \\
& \left(\mathbf{K}_{\mathbf{X}}-\mathbf{K}_{\mathbf{X Y}} \overline{\mathbf{K}}_{\mathbf{Y}}^{-1} \overline{\mathbf{K}}_{\mathbf{X Y}}^{\dagger}\left(\overline{\mathbf{K}}_{\mathbf{X Y}} \overline{\mathbf{K}}_{\mathbf{Y}}^{-1} \mathbf{K}_{\mathbf{Y}} \overline{\mathbf{K}}_{\mathbf{Y}}^{-1} \overline{\mathbf{K}}_{\mathbf{X Y}}^{\dagger}\right)^{+}\right. \\
& \left.\left.\times \overline{\mathbf{K}}_{\mathbf{X Y}} \overline{\mathbf{K}}_{\mathbf{Y}}^{-1} \mathbf{K}_{\mathbf{X Y}}^{\dagger}\right)\right) \\
+ & \max \min \left\{R+\frac{1}{2} \log \left|\mathbf{I}-\mathbf{\Omega K}_{\mathbf{X} \mid \mathbf{Y}}\right|,\right. \\
1 & \frac{1}{2} \log \mid \mathbf{I}+\mathbf{\Omega K}_{\mathbf{X Y}} \\
& \left.\times\left(\mathbf{K}_{\mathbf{Y}}^{-1}-\mathbf{K}_{\mathbf{Y}}^{-1} \overline{\mathbf{K}}_{\mathbf{Y}} \overline{\mathbf{K}}_{\mathbf{X} \mathbf{Y}}^{+} \overline{\mathbf{K}}_{\mathbf{X Y}} \overline{\mathbf{K}}_{\mathbf{Y}}^{-1}\right) \mathbf{K}_{\mathbf{X Y}}^{\dagger} \mid\right\},(14)
\end{aligned}
$$

where the maximization in the last term is over all matrices $\mathbf{0} \preceq \mathbf{\Omega} \mathbf{K}_{\mathbf{X} \mid \mathbf{Y}}^{-1}$ and where $\overline{\mathbf{K}}_{\mathbf{X Y}}^{+}$designates the MoorePenrose pseudo inverse of $\overline{\mathbf{K}}_{\mathbf{X Y}}$.

Proof: See Section IV

Remark 1. The theorem recovers the result of [4. Theorem 7] in the special case of testing against independent and $m=$ $q=1$. In this case, when the distribution $P_{X Y}$ used under the null hypothesis $\mathcal{H}=0$ describes the channel $Y=X+N$ with $X$ and $N$ independent Gaussian both with zero mean and respective variances $\sigma_{X}^{2}$ and $\sigma_{N}^{2}$, and the joint law $\bar{P}_{X Y}$ under $\mathcal{H}=1$ describes a pair of independent Gaussians of variances $\sigma_{X}^{2}+\sigma_{N}^{2}$ and $\sigma_{N}^{2}$, then:

$$
E(R)=\frac{1}{2} \log \left(\frac{\sigma_{X}^{2}+\sigma_{N}^{2}}{\sigma_{N}^{2}+2^{-2 R} \sigma_{X}^{2}}\right) .
$$

\section{Proof of TheOREM 1}

We first derive an auxiliary result. Consider a slight generalization of the discrete memoryless single-sensor singledetector hypothesis testing against conditional independence problem where the marginals are not identical under the two hypotheses. Specifically, consider the problem of Figure 2 where under

$\mathcal{H}=0: \quad\left\{\left(X_{t}, U_{t}, V_{t}\right)\right\}_{t=1}^{n}$ i.i.d. $\sim P_{X U V}$ 


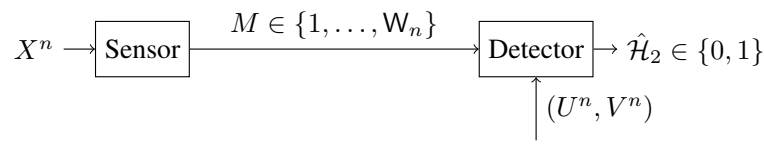

Fig. 2. Hypothesis testing with two detector observations.

$\mathcal{H}=1: \quad\left\{\left(X_{t}, U_{t}, V_{t}\right)\right\}_{t=1}^{n}$ i.i.d. $\sim \bar{P}_{X U V}=\bar{P}_{U} \bar{P}_{X \mid U} \bar{P}_{V \mid U}$.

for arbitrary distributions $P_{X U V}, \bar{P}_{U}, \bar{P}_{X \mid U}$, and $\bar{P}_{V \mid U}$.

In this new setup the message $M$ and the decision $\hat{\mathcal{H}}$ are obtained as described in the previous section if the observation $\mathbf{Y}^{n}$ is replaced by the pair of sequences $\mathbf{U}^{n} \triangleq\left(\mathbf{U}_{1}, \ldots, \mathbf{U}_{n}\right)$ and $\mathbf{V}^{n} \triangleq\left(\mathbf{V}_{1}, \ldots, \mathbf{V}_{n}\right)$. Type-I and type-II error probabilities, achievable exponents, and exponent-rate function are defined as in Section $\amalg$.

Lemma 1. If the joint distribution $P_{X U}$ under the null hypothesis satisfies

$$
P_{X U}=\underset{\substack{\tilde{P}_{X U}: \tilde{P}_{X}=P_{X} \\ \tilde{P}_{U}=P_{U}}}{\arg \min } D\left(\tilde{P}_{X U} \| \bar{P}_{X U}\right),
$$

the rate exponent function is given by

$$
\begin{aligned}
E(R)= & D\left(P_{X U} \| \bar{P}_{X U}\right)+\mathbb{E}_{P_{U}}\left[D\left(P_{V \mid U} \| \bar{P}_{V \mid U}\right)\right] \\
& +\max I(S ; V \mid U)
\end{aligned}
$$

where in (18) the maximization is over all conditionals p.m.f.s $P_{S \mid X}$ for which $I(S ; X \mid U) \leq R$.

Proof of Lemma [1 By [1, Theorem 4]:

$$
E(R)=\varliminf_{n \rightarrow \infty} E_{n}(R),
$$

where

$$
E_{n}(R) \triangleq \max _{\substack{\phi_{n} \\ \log _{2}\left|\phi_{n}\right| \leq n R}} \frac{1}{n} D\left(P_{\phi_{n}\left(X^{n}\right) U^{n} V^{n}} \| \bar{P}_{\phi_{n}\left(X^{n}\right) U^{n} V^{n}}\right) .
$$

Next, notice that by the chain rule for KL divergence, the data processing inequality, and some simple manipulations, we have

$$
\begin{aligned}
D & \left(P_{\phi_{n}\left(X^{n}\right) U^{n} V^{n}} \| \bar{P}_{\phi_{n}\left(X^{n}\right) U^{n} V^{n}}\right) \\
= & D\left(P_{\phi_{n}\left(X^{n}\right) U^{n}} \| \bar{P}_{\phi_{n}\left(X^{n}\right) U^{n}}\right) \\
& +\mathbb{E}_{P_{\phi_{n}\left(X^{n}\right) U^{n}}}\left[D\left(P_{V^{n} \mid \phi_{n}\left(X^{n}\right) U^{n}} \| \bar{P}_{V^{n} \mid U^{n}}\right)\right] \\
= & D\left(P_{\phi_{n}\left(X^{n}\right) U^{n}} \| \bar{P}_{\phi_{n}\left(X^{n}\right) U^{n}}\right)+I\left(V^{n} ; \phi_{n}\left(X^{n}\right) \mid U^{n}\right) \\
& +n \mathbb{E}_{P_{U}}\left[D\left(P_{V \mid U} \| \bar{P}_{V \mid U}\right)\right] \\
& (a) \\
\leq & n D\left(P_{X U} \| \bar{P}_{X U}\right)+I\left(V^{n} ; \phi_{n}\left(X^{n}\right) \mid U^{n}\right) \\
& +n \mathbb{E}_{P_{U}}\left[D\left(P_{V \mid U} \| \bar{P}_{V \mid U}\right)\right]
\end{aligned}
$$

where $(a)$ holds by the data-processing inequality for KLdivergence and because $X^{n}$ and $U^{n}$ are i.i.d.

We can thus bound $E(R)$ as:

$$
\begin{aligned}
E(R) \leq & D\left(P_{X U} \| \bar{P}_{X U}\right)+\mathbb{E}_{P_{U}}\left[D\left(P_{V \mid U} \| \bar{P}_{V \mid U}\right)\right] \\
& +\underline{\lim }_{n \rightarrow \infty} \max _{\substack{\phi_{n} \vdots \\
\log _{2}\left|\phi_{n}\right| \leq n R}} \frac{1}{n} I\left(V^{n} ; \phi_{n}\left(X^{n}\right) \mid U^{n}\right) .
\end{aligned}
$$

Next we use that by [1, Theorem 4] and [4, Theorem 3] both sides of

$$
\begin{aligned}
\varliminf_{n \rightarrow \infty} \max _{\substack{\phi_{n} \\
\log _{2}\left|\phi_{n}\right| \leq n R}} \frac{1}{n} \cdot\left(I\left(\phi_{n}\left(X^{n}\right) ; V^{n} \mid U^{n}\right)\right) \\
=\max _{\substack{P_{S \mid X}: \\
I(S ; X \mid U) \leq R}} I(S ; V \mid U)
\end{aligned}
$$

characterize the optimal type-II error exponent of a hypothesis testing against conditional independence problem at rate $R$, and thus coincide.

Combining (19) and (25) we obtain:

$$
\begin{aligned}
E(R) & \leq D\left(P_{X U} \| \bar{P}_{X U}\right)+\mathbb{E}_{P_{U}}\left[D\left(P_{V \mid U} \| \bar{P}_{V \mid U}\right)\right] \\
& +\max I(S ; V \mid U) .
\end{aligned}
$$

The reverse inequality follows from the achievable typeII error exponent of Shimokawa-Han-Amari (SHA) [3] (see [22, Section IV] for an analysis) which states that for every choice of the conditional distribution $P_{S \mid X}$ satisfying $R \geq$ $I(S ; X \mid U, V)$ the following lower bound holds:

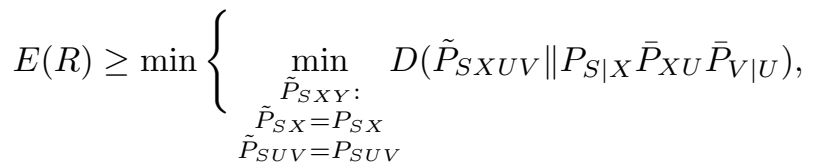

$$
\begin{aligned}
& \min _{\tilde{P}_{S X U}} D\left(\tilde{P}_{S X U V} \| P_{S \mid X} \bar{P}_{X U} \bar{P}_{V \mid U}\right) \\
& \tilde{P}_{S X U V} \text { : } \\
& \tilde{P}_{S X}=P_{S X} \\
& \begin{array}{c}
\tilde{P}_{U V}=P_{U V} \\
H(S \mid U V) \leq H_{\tilde{P}_{S U V}}
\end{array}(S \mid U V) \\
& +R-I(S ; X \mid U V)\}
\end{aligned}
$$

where the mutual information $I(S ; X \mid U V)$ is calculated according to $P_{S \mid X} P_{U V X}$. In what follows we show that the SHA result implies that the error exponent on the RHS of (18) is achievable. As in [4], we restrict to distributions $P_{S \mid X}$ satisfying

$$
R \geq I(S ; X \mid U)
$$

and drop the condition $H(S \mid U, V) \leq H_{\tilde{P}_{S U V}}(S \mid U, V)$ in the minimization. These changes can lead to a smaller exponent than in [3], and thus the resulting exponent is still achievable.

By the Markov chain $S \odot X-(U, V)$, Condition 28 implies

$$
R-I(S ; X \mid U, V) \geq I(S ; V \mid U) .
$$

Moreover, by the chain rule and the nonnegativity and convexity of KL divergence, for any $\tilde{P}_{S X U V}$ :

$$
\begin{aligned}
& D\left(\tilde{P}_{S X U V} \| P_{S \mid X} \bar{P}_{X U} \bar{P}_{V \mid U}\right) \\
& \quad \geq D\left(\tilde{P}_{X U V} \| \bar{P}_{X U} \bar{P}_{V \mid U}\right) \\
& \quad=D\left(\tilde{P}_{X U} \| \bar{P}_{X U}\right)+\mathbb{E}_{\tilde{P}_{X U}}\left[D\left(\tilde{P}_{V \mid X U} \| \bar{P}_{V \mid U}\right)\right] \\
& \geq D\left(\tilde{P}_{X U} \| \bar{P}_{X U}\right)+\mathbb{E}_{\tilde{P}_{U}}\left[D\left(\tilde{P}_{V \mid U} \| \bar{P}_{V \mid U}\right)\right] .
\end{aligned}
$$

By (29) and (32) and since the second minimization in (27) is over distributions $\tilde{P}_{S X U V}$ satisfying $\tilde{P}_{U V}=P_{U V}$, we conclude that under conditions (17) and (28) the second term in 27) is lower bounded by

$$
\theta \triangleq D\left(P_{X U} \| \bar{P}_{X U}\right)+\mathbb{E}_{P_{U}}\left[D\left(P_{V \mid U} \|\left.\bar{P}\right|_{V \mid U}\right)\right]+I(S ; V \mid U) .
$$


We now lower bound the first term in 27). By the chain rule and the nonnegativity and convexity of KL divergence, for any $\tilde{P}_{S X U V}$ where $\tilde{P}_{S X}=P_{S \mid X} P_{X}$ :

$$
\begin{aligned}
& D\left(\tilde{P}_{S X U V} \| P_{S \mid X} \bar{P}_{X U} \bar{P}_{V \mid U}\right) \\
& \quad=D\left(\tilde{P}_{X U} \| \bar{P}_{X U}\right)+\mathbb{E}_{\tilde{P}_{X U}}\left[D\left(\tilde{P}_{S V \mid X U} \| P_{S \mid X} \bar{P}_{V \mid U}\right)\right] \\
& \geq D\left(\tilde{P}_{X U} \| \bar{P}_{X U}\right)+\mathbb{E}_{\tilde{P}_{U}}\left[D\left(\tilde{P}_{S V \mid U} \| P_{S} \bar{P}_{V \mid U}\right)\right]
\end{aligned}
$$

Where in the last inequality we used that $\sum_{x} \tilde{P}_{X}(x) P_{S \mid X}(s \mid x)=P_{S}(s)$ because $\tilde{P}_{X}=P_{X}$. We now notice that the first miniminization in (27) is only over distributions $\tilde{P}_{S X U V}$ satisfying $\tilde{P}_{S U V}=P_{S U V}$ and therefore:

$$
\begin{aligned}
\mathbb{E}_{\tilde{P}_{U}}[ & \left.D\left(\tilde{P}_{S V \mid U} \| P_{S} \bar{P}_{V \mid U}\right)\right] \\
& =\mathbb{E}_{P_{U}}\left[D\left(P_{S V \mid U} \| P_{S} \bar{P}_{V \mid U}\right)\right] \\
& =I(S ; V \mid U)+\mathbb{E}_{P_{U}}\left[D\left(P_{V \mid U} \| \bar{P}_{V \mid U}\right)\right] .
\end{aligned}
$$

Combining (34) and (36), we conclude that under Condition (17) also the first term in the minimization in 27) is lower bounded by $\theta$. This establishes the achievability of the righthand side of (18).

We turn to the proof of Theorem 1 Define

$$
\begin{aligned}
\mathbf{U} & =\mathbb{E}_{\bar{P}}[\mathbf{X} \mid \mathbf{Y}] \\
\mathbf{V} & =\mathbf{Y}
\end{aligned}
$$

and notice that under $\mathcal{H}=1$ they satisfy the Markov chain

$$
\mathbf{X} \odot \mathbf{U} \odot \mathbf{V} \text {. }
$$

In what remains, we assume that instead of $\mathbf{Y}^{n}$ the decoder observes the pair of sequences $\left(\mathbf{U}^{n}, \mathbf{V}^{n}\right)$ which is i.i.d according to the joint distribution of $(\mathbf{U}, \mathbf{V})$. This new system is depicted in Figure 2. Since there is a bijection between $\mathbf{Y}^{n}$ and $\left(\mathbf{U}^{n}, \mathbf{V}^{n}\right)$, the error exponent of the new system coincides with the error exponent of the original system. Moreover, by the Markov chain (39) the new system is a generalized testing against conditional independence problem as described in (16). We next argue that under condition $C$ in Theorem 1 and because $P_{\mathbf{X}^{n}} \mathbf{U}^{n} \mathbf{V}^{n}$ and $\bar{P}_{\mathbf{X}^{n}} \mathbf{U}^{n} \mathbf{V}^{n}$ are multivariate Gaussian distributions, the new system also satisfies Condition (17) in Lemma 1. The optimal exponent $E(R)$ will then follow immediately from this Lemma 1 .

To show that for multivariate Gaussian distributions $P_{\mathbf{X}^{n}} \mathbf{U}^{n} \mathbf{V}^{n}$ and $\bar{P}_{\mathbf{X}^{n} \mathbf{U}^{n} \mathbf{V}^{n}}$ condition $\mathrm{C}$ in (11) implies (17), we first show that under this Gaussian assumption the minimizer of

$$
\underset{\substack{\mathbf{X}_{\mathbf{U}}: \tilde{P}_{\mathbf{X}}=P_{\mathbf{X}} \\ \tilde{P}_{\mathbf{U}}=P_{\mathbf{U}}}}{\arg \min } D\left(\tilde{P}_{\mathbf{X} \mathbf{U}} \| \bar{P}_{\mathbf{X} \mathbf{U}}\right)
$$

is a multivariate Gaussian distribution. To see this fix any distribution $\tilde{P}_{\mathbf{X U}}$ with $\tilde{P}_{\mathbf{X}}=P_{\mathbf{X}}$ and $\tilde{P}_{\mathbf{U}}=P_{\mathbf{U}}$ and let $\tilde{P}_{\mathbf{X U}}^{G}$ be a multivariate Gaussian distribution with same covariance matrix as $\tilde{P}_{\mathrm{XU}}$. Then:

$$
\begin{aligned}
D\left(\tilde{P}_{\mathbf{X U}} \| \bar{P}_{\mathbf{X U}}\right) & =-h\left(\tilde{P}_{\mathbf{X U}}\right)-\mathbb{E}_{\tilde{P}}\left[\log \bar{P}_{\mathbf{X U}}\right] \\
& \geq-h\left(\tilde{P}_{\mathbf{X U}}^{G}\right)-\mathbb{E}_{\tilde{P}}\left[\log \bar{P}_{\mathbf{X U}}\right] \\
& =-h\left(\tilde{P}_{\mathbf{X U}}^{G}\right)-\mathbb{E}_{\tilde{P}^{G}}\left[\log \bar{P}_{\mathbf{X U}}\right],
\end{aligned}
$$

where the inequality holds because a Gaussian distribution maximizes differential entropy under a fixed covariance matrix constraint and where the last equality holds because
$\mathbb{E}\left[\log \bar{P}_{U X}\right]$ only depends on the covariance matrix of $(U, X)$ which is the same under $\tilde{P}$ and $\tilde{P}^{G}$. By straightforward algebra, it can then be shown that if condition $C$ in (11) holds, then $P_{\mathbf{U X}}$ is the multivariate Gaussian distribution that minimizes (17).

We conclude that the optimal exponent $E(R)$ is given by (18) in Lemma 1 . We evaluate (18) for our problem. For simplicity, we rewrite

$$
\begin{aligned}
& D\left(P_{\mathbf{X U}} \| \bar{P}_{\mathbf{X U}}\right)+\mathbb{E}_{P_{\mathbf{U}}}\left[D\left(P_{\mathbf{V} \mid \mathbf{U}} \| \bar{P}_{\mathbf{V} \mid \mathbf{U}}\right)\right. \\
& \quad=D\left(P_{\mathbf{U V}} \| \bar{P}_{\mathbf{U V}}\right)+\mathbb{E}_{P_{\mathbf{U}}}\left[D\left(P_{\mathbf{X} \mid \mathbf{U}} \| \bar{P}_{\mathbf{X} \mid \mathbf{U}}\right)\right]
\end{aligned}
$$

and proceed to compute

$$
D\left(P_{\mathbf{U V}} \| \bar{P}_{\mathbf{U V}}\right)=\frac{q}{2}+\frac{1}{2} \log \frac{\left|\overline{\mathbf{K}}_{\mathbf{Y}}\right|}{\left|\overline{\mathbf{K}}_{\mathbf{Y}}\right|}+\frac{1}{2} \operatorname{Tr}\left(\overline{\mathbf{K}}_{\mathbf{Y}}^{-1} \mathbf{K}_{\mathbf{Y}}\right)
$$

and

$$
\begin{aligned}
D( & \left.P \mathbf{X}|| \bar{P}_{\mathbf{X}} \mid \mathbf{U}\right)=\frac{m}{2}+\frac{1}{2} \log \left|\overline{\mathbf{K}}_{\mathbf{X} \mid \mathbf{Y}}\right|-\log \mid \mathbf{K}_{\mathbf{X}}-\mathbf{K}_{\mathbf{X Y}} \overline{\mathbf{K}}_{\mathbf{Y}}^{-1} \\
& \times \overline{\mathbf{K}}_{\mathbf{X Y}}^{\dagger}\left(\overline{\mathbf{K}}_{\mathbf{X Y}} \overline{\mathbf{K}}_{\mathbf{Y}}^{-1} \mathbf{K}_{\mathbf{Y}} \overline{\mathbf{K}}_{\mathbf{Y}}^{-1} \overline{\mathbf{K}}_{\mathbf{X Y}}^{\dagger}\right)^{+} \overline{\mathbf{K}}_{\mathbf{X Y}} \overline{\mathbf{K}}_{\mathbf{Y}}^{-1} \mathbf{K}_{\mathbf{X Y}}^{\dagger} \mid \\
+ & \frac{1}{2} \operatorname{Tr}\left(\overline{\mathbf{K}}_{\mathbf{X} \mid \mathbf{Y}}^{-1} \times\right. \\
& \left(\mathbf{K}_{\mathbf{X}}-\mathbf{K}_{\mathbf{X Y}} \overline{\mathbf{K}}_{\mathbf{Y}}^{-1} \overline{\mathbf{K}}_{\mathbf{X Y}}^{\dagger}\left(\overline{\mathbf{K}}_{\mathbf{X Y}} \overline{\mathbf{K}}_{\mathbf{Y}}^{-1} \mathbf{K}_{\mathbf{Y}} \overline{\mathbf{K}}_{\mathbf{Y}}^{-1} \overline{\mathbf{K}}_{\mathbf{X Y}}^{\dagger}\right)^{+}\right. \\
& \left.\left.\times \overline{\mathbf{K}}_{\mathbf{X Y}} \overline{\mathbf{K}}_{\mathbf{Y}}^{-1} \mathbf{K}_{\mathbf{X Y}}^{\dagger}\right)\right) .
\end{aligned}
$$

It remains to find $\max I(S ; \mathbf{Y} \mid \mathbf{U})$ where the maximum is over all test channels $P_{S \mid \mathbf{X}}$ satisfying $I(S ; \mathbf{X} \mid \mathbf{U}) \leq R$. Let $\tilde{U}=U+\epsilon Z$ where $Z \sim(0, I)$. Applying the result of [5, Theorem 2] on the triple $(X, Y, \tilde{U})$, which is Gaussian, and then taking the limit $\epsilon \rightarrow 0$ we get:

$$
\begin{aligned}
& \max _{\substack{P_{S \mid \mathbf{X}}: \\
I(S ; \mathbf{X} \mid \mathbf{U}) \leq R}} I(S ; \mathbf{Y} \mid \mathbf{U})=\max \min \left\{R+\frac{1}{2} \log \left|\mathbf{I}-\mathbf{\Omega K}_{\mathbf{X} \mid \mathbf{Y}}\right| ;\right. \\
& \quad \frac{1}{2} \log \mid \mathbf{I}+\mathbf{\Omega K}_{\mathbf{X Y}} \\
& \left.\quad \times\left(\mathbf{K}_{\mathbf{Y}}^{-1}-\mathbf{K}_{\mathbf{Y}}^{-1} \overline{\mathbf{K}}_{\mathbf{Y}} \overline{\mathbf{K}}_{\mathbf{X} \mathbf{Y}}^{+} \overline{\mathbf{K}}_{\mathbf{X Y}} \overline{\mathbf{K}}_{\mathbf{Y}}^{-1}\right) \mathbf{K}_{\mathbf{X Y}}^{\dagger} \mid\right\},
\end{aligned}
$$

where the maximization in the last term is over all matrices $\mathbf{0} \preceq \mathbf{\Omega} \mathbf{K}_{\mathbf{X} \mid \mathbf{Y}}^{-1}$ and where $\overline{\mathbf{K}}_{\mathbf{X Y}}^{+}$designates the MoorePenrose pseudo inverse of $\overline{\mathbf{K}}_{\mathbf{X Y}}$.

Summing (43)-(45) we obtain the desired result in (14), which completes the proof of Theorem 1

\section{Discussion}

In what follows, we show that constraint $\mathrm{C}$ as given by 11 is fulfilled for a large class of sources even when $m=1$ and $q=2$. Let $X$ be a scalar source that is observed at the sensor and $\mathbf{Y}=\left(Y_{1}, Y_{2}\right)$ a 2 -dimensional source that is observed at the detector. For convenience, let

$$
\mathbf{K}=\left[\begin{array}{ccc}
\sigma_{X}^{2} & \sigma_{X Y_{1}} & \sigma_{X Y_{2}} \\
\sigma_{X Y_{1}} & \sigma_{Y_{1}}^{2} & \sigma_{Y_{1} Y_{2}} \\
\sigma_{X Y_{2}} & \sigma_{Y_{1} Y_{2}} & \sigma_{Y_{2}}^{2}
\end{array}\right] \text { and } \overline{\mathbf{K}}=\left[\begin{array}{ccc}
\bar{\sigma}_{X}^{2} & \bar{\sigma}_{X Y_{1}} & \bar{\sigma}_{X Y_{2}} \\
\bar{\sigma}_{X Y_{1}} & \bar{\sigma}_{Y_{1}}^{2} & \bar{\sigma}_{Y_{1} Y_{2}} \\
\bar{\sigma}_{X Y_{2}} & \bar{\sigma}_{Y_{1} Y_{2}} & \bar{\sigma}_{Y_{2}}^{2}
\end{array}\right] \text {. }
$$


Also, let

$$
a=\left(\bar{\sigma}_{X Y_{2}} \bar{\sigma}_{Y_{1} Y_{2}}-\bar{\sigma}_{X Y_{1}} \bar{\sigma}_{Y_{2}}^{2}\right) \text { and } b=\left(\bar{\sigma}_{X Y_{1}} \bar{\sigma}_{Y_{1} Y_{2}}-\bar{\sigma}_{X Y_{2}} \bar{\sigma}_{Y_{1}}^{2}\right) \text {. }
$$

For this example the constraint $\mathrm{C}$ as given by (11) reduces to

$$
\begin{aligned}
\text { i) } & \sigma_{X}^{2}=\bar{\sigma}_{X}^{2}, \\
\text { ii) } & a\left(\sigma_{X Y}-\bar{\sigma}_{X Y}\right)+b\left(\sigma_{X Z}-\bar{\sigma}_{X Z}\right)=0 \\
\text { iii) } & a^{2}\left(\sigma_{Y}^{2}-\bar{\sigma}_{Y}^{2}\right)+2 a b\left(\sigma_{Y Z}-\bar{\sigma}_{Y Z}\right)+b^{2}\left(\sigma_{Z}^{2}-\bar{\sigma}_{Z}^{2}\right)=0
\end{aligned}
$$

For example, if all components have unit variance under both $P$ and $\bar{P}$, i.e., $\sigma_{X}^{2}=\sigma_{Y_{1}}^{2}=\sigma_{Y_{2}}^{2}=1$ and $\bar{\sigma}_{X}^{2}=\bar{\sigma}_{Y_{1}}^{2}=$ $\bar{\sigma}_{Y_{2}}^{2}=1$ then all definite positive matrices $\mathbf{K}$ and $\overline{\mathbf{K}}$ of the form

$$
\mathbf{K}=\left[\begin{array}{ccc}
1 & a_{12} & h\left(\bar{a}_{12}, \bar{a}_{13}, \bar{a}_{23}, a_{12}\right) \\
a_{12} & 1 & \bar{a}_{23} \\
h\left(\bar{a}_{12}, \bar{a}_{13}, \bar{a}_{23}, a_{12}\right) & \bar{a}_{23} & 1
\end{array}\right],
$$

and

$$
\overline{\mathbf{K}}=\left[\begin{array}{ccc}
1 & a_{12} & \bar{a}_{13} \\
a_{12} & 1 & \bar{a}_{23} \\
\bar{a}_{13} & \bar{a}_{23} & 1
\end{array}\right]
$$

for some arbitrary parameters $a_{12}, \bar{a}_{12}, \bar{a}_{13}, \bar{a}_{23}$, satisfy the constraint (47). Here

$$
h\left(x, y_{1}, y_{2}, t\right)=y_{1}-\left(t-y_{2}\right) \frac{y_{1} y_{2}-x}{x y_{2}-y_{1}} .
$$

Example 1. Let

$$
\mathbf{K}=\left[\begin{array}{ccc}
1 & 0.4 & \alpha \\
0.4 & 1 & 0.1 \\
\alpha & 0.1 & 1
\end{array}\right] \text { and } \quad \overline{\mathbf{K}}=\left[\begin{array}{ccc}
1 & 0.1 & -0.8 \\
0.1 & 1 & 0.1 \\
-0.8 & 0.1 & 1
\end{array}\right] \text {, }
$$

with $\alpha \approx-0.73333$. It is easy to see that 477 is fulfilled. Figure 3 shows the evolution of the optimal exponent $E$ as a function of the communication rate $R$ as given by Theorem 1 for this example. Notice that Han's exponent [2. Theorem 2] is strictly suboptimal for this example?.

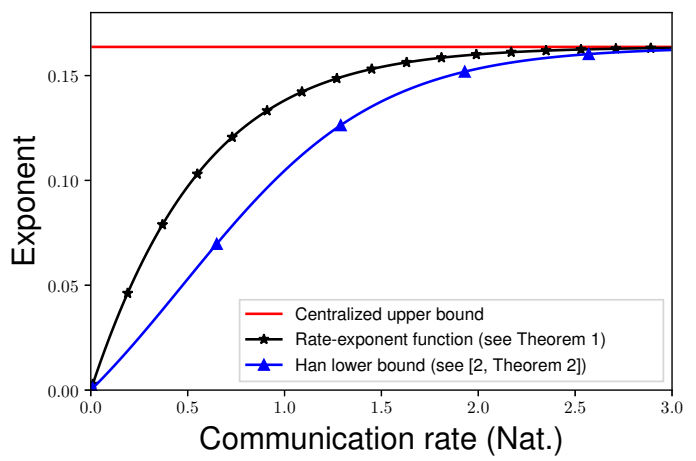

Fig. 3. Rate-exponent region for Example 1

\section{ACKNOWLEDGEMENT}

The work of M. Wigger was funded by the European Research Council (ERC) under the European Union's Horizon 2020 under grant agreement No 715111.

\footnotetext{
${ }^{1}$ In the figure, Han's exponent as given by [2] Theorem 2] is computed using Gaussian test channels $P_{U \mid X}$ and Gaussian $\tilde{U}$
}

\section{REFERENCES}

[1] R. Ahlswede and I. Csiszar, "Hypothesis testing with communication constraints," IEEE Trans. Inf. Theory, vol. 32, no. 4, pp. 533-542, July 1986.

[2] T. S. Han, "Hypothesis testing with multiterminal data compression," IEEE Trans. Inf. Theory, vol. 33, no. 6, pp. 759-772, November 1987.

[3] H. Shimokawa, T. S. Han, and S. Amari, "Error bound of hypothesis testing with data compression," in Proc. IEEE ISIT'94, Jun. 1994, p. 114.

[4] M. S. Rahman and A. B. Wagner, "On the optimality of binning for distributed hypothesis testing," IEEE Trans. Inf. Theory, vol. 58, no. 10, pp. 6282-6303, Oct. 2012.

[5] A. Zaidi and I. E. Aguerri, "Optimal rate-exponent region for a class of hypothesis testing against conditional independence problems," in 2019 IEEE Information Theory Workshop (ITW), Aug 2019, pp. 1-5.

[6] W. Zhao and L. Lai, "Distributed testing against independence with multiple terminals," in 2014 52nd Annual Allerton Conference on Communication, Control, and Computing (Allerton), Sep. 2014, pp. 1246-1251.

[7] S. Salehkalaibar, M. Wigger, and R. Timo, "On hypothesis testing against conditional independence with multiple decision centers," IEEE Transactions on Communications, vol. 66, no. 6, pp. 2409-2420, June 2018.

[8] P. Escamilla, M. Wigger, and A. Zaidi, "Distributed hypothesis testing: cooperation and concurrent detection," in revision for publication in the IEEE Transactions of Information Theory, 2019.

[9] C. Tian and J. Chen, "Successive refinement for hypothesis testing and lossless one-helper problem," IEEE Transactions on Information Theory, vol. 54, no. 10, pp. 4666-4681, Oct 2008.

[10] Y. Xiang and Y. H. Kim, "Interactive hypothesis testing with communication constraints," in Proc. of Allerton Conference on Comm., Control, and Comp., Monticello (IL), USA, Oct. 2012, pp. 1065-1072.

[11] G. Katz, P. Piantanida, and M. Debbah, "Collaborative distributed hypothesis testing with general hypotheses," in 2016 IEEE International Symposium on Information Theory (ISIT), July 2016, pp. 1705-1709.

[12] M. Wigger and R. Timo, "Testing against independence with multiple decision centers," in 2016 International Conference on Signal Processing and Communications (SPCOM), Bangalore, India, Jun. 2016, pp. $1-5$.

[13] S. Salehkalaibar, M. A. Wigger, and L. Wang, "Hypothesis testing in multi-hop networks," arXiv:1708.05198, 2017.

[14] P. Escamilla, M. Wigger, and A. Zaidi, "Distributed hypothesis testing with concurrent detections," in 2018 IEEE International Symposium on Information Theory (ISIT), June 2018, pp. 166-170.

[15] W. Zhao and L. Lai, "Distributed testing with cascaded encoders," IEEE Transactions on Information Theory, vol. 64, no. 11, pp. 7339-7348, Nov 2018

[16] P. Escamilla, A. Zaidi, and M. Wigger, "Distributed hypothesis testing with collaborative detection," in 2018 56th Annual Allerton Conference on Communication, Control, and Computing (Allerton), Oct 2018, pp. 512-518.

[17] S. Salehkalaibar and M. Wigger, "Distributed hypothesis testing over multi-access channels," in 2018 Information Theory and Applications Workshop (ITA), Feb 2018, pp. 1-5.

[18] S. Sreekumar and D. Gündüz, "Distributed hypothesis testing over noisy channels," in 2017 IEEE International Symposium on Information Theory (ISIT), June 2017, pp. 983-987.

[19] J. Liao, L. Sankar, F. P. Calmon, and V. Y. Tan, "Hypothesis testing under maximal leakage privacy constraints," in 2017 IEEE International Symposium on Information Theory (ISIT). IEEE, 2017, pp. 779-783.

[20] J. Liao, L. Sankar, V. Y. F. Tan, and F. du Pin Calmon, "Hypothesis testing under mutual information privacy constraints in the high privacy regime," IEEE Transactions on Information Forensics and Security, vol. 13, no. 4, pp. 1058-1071, April 2018

[21] S. Sreekumar, D. Gündüz, and A. Cohen, "Distributed hypothesis testing under privacy constraints," in 2018 IEEE Information Theory Workshop (ITW), Nov 2018, pp. 1-5.

[22] S. Salehkalaibar, M. Wigger, and L. Wang, "Hypothesis testing over the two-hop relay network," IEEE Transactions on Information Theory, vol. 65 , no. 7, pp. 4411-4433, July 2019. 